\title{
Autopercepção do consumo alimentar e adesão aos Dez Passos para Alimentação Saudável entre universitários de Porto Alegre, Brasil
}

\author{
Self-perception of food consumption and observance of the Ten \\ Steps to Healthy Eating among university students in Porto Alegre, \\ Brazil
}

Rosenir Korpalski de Souza (https://orcid.org/0000-0001-5816-3636) ${ }^{1}$

Vanessa Backes (https://orcid.org/0000-0002-3860-7866) ${ }^{2}$

${ }^{1}$ Centro de Pesquisa Clínica, Hospital de Clínicas de Porto Alegre. R. Jacinto Gomes 543, apto 302, Bairro Santana. 90040-270 Porto Alegre RS Brasil. rosenirks@gmail.com

${ }^{2}$ Universidade do Vale do

Rio dos Sinos. São Leopoldo RS Brasil.

\begin{abstract}
The scope of this study is to analyze the relationship between self-perception of eating habits and observance of the Ten Steps to Adequate and Healthy Eating among university students in Porto Alegre/RS. An online questionnaire was applied to students at a private university in Porto Alegre. Personal information, anthropometric data and eating behavior of the participants were collected. Data were analyzed by frequency and proportion, and statistical associations using Pearson's Chi-square and the linear trend test, adopting a significance level of 5\%, using version 23.0 of the SPSS statistical program. The sample consisted of 357 students, $61.3 \%$ of which were female, between 20 and 29 years of age (56\%). A total of $34.2 \%$ of the students were overweight and $55.5 \%$ did not consider they had healthy eating habits. Low observance was observed in eight of the Ten Steps to Adequate and Healthy Eating A review of healthy eating revealed an inverse relationship with observance of the guideline steps, since only 3 steps were followed by more than $60 \%$ of the students who evaluated their own eating habits positively. This result suggests that this population may have a distorted view of their own eating habits and need to be enlightened regarding adequate and healthy eating.
\end{abstract}

Key words Self-perception, Healthy eating, Eating behavior
Resumo O objetivo deste estudo é analisar a relação entre a autopercepção alimentar e a adesão aos Dez Passos para uma Alimentação Adequada e Saudável entre estudantes universitários de Porto Alegre/RS. Um questionário online foi aplicado com estudantes de uma universidade privada de Porto Alegre. Foram coletados dados pessoais, antropométricos e do comportamento alimentar dos participantes. Os dados foram analisados através de frequência e proporção e as associações estatísticas pelo do teste do Qui-quadrado de Pearson e de tendência linear, adotando-se um nivel de significância de 5\%, utilizando-se o programa estatístico SPSS versão 23.0. A amostra foi composta por 357 alunos, sendo 61,3\% do sexo feminino, com idade entre 20 e 29 anos (56\%). 34,2\% dos alunos apresentaram excesso de peso e 55,5\% não consideraram a alimentação saudável. Observouse baixa adesão a oito dos Dez Passos para uma Alimentação Adequada e Saudável. Considerar a alimentação saudável demonstrou relação inversa com a adesão aos passos do Guia Alimentar, pois apenas 3 passos foram seguidos por mais de 60\% dos alunos que avaliaram positivamente a própria alimentação. Esse resultado sugere que essa população pode possuir uma visão distorcida da própria alimentação e precisa ser esclarecida quanto à alimentação adequada e saudável.

Palavras-chave Autopercepção, Alimentação saudável, Comportamento alimentar 


\section{Introdução}

Muitos são os fatores que constroem e modificam o comportamento alimentar. Influências externas e internas estão intimamente ligadas com a relação entre o indivíduo e a forma como ele se alimenta ${ }^{1}$. Muitas dessas influências podem conduzir a um comportamento alimentar inadequado e consequentemente ao excesso de peso, fator capaz de desencadear o desenvolvimento de doenças crônicas não transmissíveis $(\mathrm{DCNT})^{2}$. Esse cenário tem sido ainda mais preocupante à medida que estudantes universitários demonstram uma crescente inadequação alimentar, bem como altos índices de excesso de peso ${ }^{3,4}$. Para prevenir as DCNT é necessário adotar um estilo de vida saudável que inclui um comportamento alimentar adequado ${ }^{5}$.

A alimentação saudável baseia-se no consumo de alimentos variados, de uma dieta equilibrada de acordo com as necessidades nutricionais individuais e de forma que esteja em harmonia com o meio cultural e social ao qual o indivíduo está inserido ${ }^{6}$. Como instrumento de orientação para alimentação saudável, o Ministério da Saúde publicou em 2014, o novo Guia Alimentar para a População Brasileira, que aborda a alimentação saudável através de diversos aspectos além do ato de consumir. As recomendações do Guia Alimentar foram sintetizadas de uma forma didática por meio dos Dez Passos para uma Alimentação Adequada e Saudável que preconizam: utilizar como base da alimentação os alimentos in natura ou minimamente processados; moderação na adição de óleos, gorduras, sal e açúcar nas preparações culinárias; limitar o consumo de alimentos processados e evitar o consumo de alimentos ultraprocessados; desenvolver e partilhar habilidades culinárias; planejar o uso do tempo para dar a alimentação o espaço que ela merece; preferir locais tranquilos e companhia na hora de fazer as refeições; procurar não fazer grandes intervalos entre refeições; optar por locais que ofereçam refeições feitas na hora ao alimentar-se fora de casa; ao fazer as compras, procurar locais que ofereçam variedade de alimentos in natura ou minimamente processados, como feiras agroecológicas, por exemplo, e ser crítico quanto à informações relacionadas à alimentação e nutrição veiculadas pela publicidade ${ }^{6}$.

No entanto, muitas dificuldades são enfrentadas para motivar mudanças em relação ao comportamento alimentar inadequado. Alguns autores sugerem a autopercepção do consumo alimentar como fator relacionado à resistência a adoção de uma alimentação saudável, visto que $\mathrm{o}$ indivíduo precisa reconhecer a necessidade de mudança para que ela possa ocorrer ${ }^{7-9}$. Sendo assim, o presente estudo tem como objetivo analisar a relação entre a autopercepção do consumo alimentar e a adesão aos Dez Passos para uma Alimentação Adequada e Saudável entre estudantes universitários de Porto Alegre, Rio Grande do Sul (RS).

\section{Métodos}

O presente estudo trata-se de uma metodologia quantitativa com delineamento transversal. Ele foi realizado com estudantes de uma universidade privada de Porto Alegre-RS, por amostra de conveniência, adotando-se um nível de significância de 5\%. Foram incluídos no estudo todos os universitários que possuíam 18 anos ou mais. Foram excluídos do estudo os alunos do curso de nutrição.

Foram coletados dados através de um questionário eletrônico que investigou nome, idade, sexo, estado civil, curso e semestre que o estudante estava cursando no momento da pesquisa. $\mathrm{O}$ questionário incluiu também questões relacionadas à saúde, como peso e altura, possuir ou não doenças, autopercepção da alimentação, bem como questões voltadas ao comportamento e ao consumo alimentar conforme preconizam os Dez Passos para Alimentação Adequada e Saudável ${ }^{6}$.

O questionário foi pré-testado com 20 estudantes no semestre anterior e após, foram realizadas as correções necessárias. Durante os meses de agosto a dezembro de 2017 foram coletados os e-mails dos alunos e enviados os questionários. Para a coleta dos e-mails dos estudantes foram realizadas abordagens nos espaços de convivência do campus e nas salas de aula, informando sobre o objetivo do estudo e convidando para responder ao questionário eletrônico. Os estudantes que aceitaram participar da pesquisa forneceram seus endereços eletrônicos e receberam um e-mail com o link do formulário a ser respondido.

Os cursos foram agrupados de acordo com as áreas do conhecimento classificadas pelo Conselho Nacional de Desenvolvimento Científico e Tecnológico $(\mathrm{CNPQ})^{10}$. Devido a classificação do CNPQ não abranger os cursos de moda, jogos digitais, publicidade e propaganda, marketing e design de produto, estes foram classificados de acordo com o site da universidade (Indústria Criativa). Para classificação do semestre optouse por dividir em duas categorias, sendo de pri- 
meiro a quarto semestre como início do curso e a partir do quinto semestre como final do curso.

Os dados antropométricos coletados foram peso e altura autorreferidos. A classificação antropométrica foi realizada através do índice de Massa Corporal (IMC), calculado mediante a equação: peso dividido pela altura ao quadrado, segundo os critérios da Organização Mundial da Saúde ${ }^{11}$. Para analisar as variáveis classificação antropométrica e adesão aos passos, optou-se por agrupar a classificação antropométrica em duas categorias. Em função do baixo número de participantes com desnutrição, estes foram agrupados aos eutróficos na categoria sem excesso de peso e sobrepeso e obesidade na categoria excesso de peso. Além disso, foram avaliadas doenças existentes.

Para avaliar a percepção do estudante diante da própria alimentação foram realizadas as perguntas: "Você considera sua alimentação saudável?" e "Você acredita que precisa realizar mudanças na sua alimentação?”. Para ambas as perguntas as opções de resposta foram: sim ou não. A fonte de conhecimento nutricional foi avaliada através da pergunta: "Quando você quer saber algo relacionado à nutrição, onde você busca a informação?". A adesão aos Dez passos para uma Alimentação Adequada e Saudável foi avaliada a partir de 15 questões baseadas nas recomendações dos Dez Passos para Alimentação Adequada e Saudável do Guia Alimentar ${ }^{6}$. As perguntas abordaram a frequência semanal de consumo de determinados alimentos, assim como aspectos pontuais do comportamento alimentar conforme descrito no Quadro 1. Devido a necessidade de contemplar o conteúdo de todos os passos, foi necessário atribuir a alguns mais de uma questão. Para considerar que o passo foi seguido o estudante deveria demonstrar adesão a todas as questões atribuídas ao passo.

Todas as perguntas do questionário de comportamento alimentar ofereceram opções fechadas de resposta. Para a questão atribuída ao passo 3 por exemplo, que preconiza o consumo de alimentos processados com moderação, foram oferecidas quatro opções de resposta: nenhuma vez por semana, uma ou duas vezes por semana, três ou quatro vezes por semana ou todos os dias. Sendo assim, adotou-se como adesão, consumir cereais integrais como pães e biscoitos quatro vezes ou menos por semana.

Com relação ao passo 5 , contemplando o aspecto comer com regularidade, atribuiu-se à questão, realizar no mínimo 3 refeições por dia. Apesar de o Guia Alimentar não apresentar essa recomendação ele apresenta por intermédio de imagens, as 3 principais refeições do dia, café da manhã, almoço e jantar, com opções de combinações de alimentos saudáveis ${ }^{6}$.

Optou-se por utilizar como parâmetro para baixa adesão aos passos do Guia Alimentar, uma frequência de adesão inferior a 60\%. Apesar de não ser um parâmetro validado, o ponto de corte de $60 \%$ é amplamente utilizado para determinar se algo ou alguém atingiu o resultado desejado $^{12-14}$.

Os dados coletados foram digitados em planilha Excel e após, exportados para o programa estatístico SPSS versão 23.0. A descrição dos participantes e a adesão aos dez passos foram analisadas através de frequência e proporção e as associações estatísticas por meio do teste do Qui-quadrado de Pearson e de tendência linear. Foram consideradas associações estatisticamente significativas aquelas com $\mathrm{p}<0,05$.

O estudo foi aprovado pelo Comitê de Ética da universidade onde o estudo foi realizado, e o Termo de Consentimento Livre e Esclarecido foi assinado de forma digital, sendo parte integrante do questionário.

\section{Resultados}

Foram coletados dados de 374 universitários, o que representa um percentual de $26,9 \%$ do total de alunos matriculados no semestre anterior ao estudo ( $n=1324)$. O percentual de exclusão foi de 4,5\% devido à idade do estudante ser inferior a 18 anos $(\mathrm{n}=14)$, por serem alunos do curso de nutrição $(\mathrm{n}=2)$ e por ter respondido ao pré-teste do questionário no semestre anterior $(\mathrm{n}=1)$, o que totalizou uma amostra de 357 estudantes.

Os dados sociodemográficos, classificação antropométrica e de saúde estão descritos na Tabela 1. A maioria dos participantes era do sexo feminino $(61,3 \% ; n=219)$, solteiro $(84 \%)$, com idade variando de 18 a 67 anos. As áreas do conhecimento com maior número de alunos foram ciências humanas $(27,2 \% \mathrm{n}=97)$ e ciências da saúde $(22,1 \% \mathrm{n}=79)$ e $62,5 \%$ dos alunos estavam no início do curso no momento da pesquisa conforme a categoria semestre. Entre os avaliados $34,2 \%$ estavam com excesso de peso, sendo $23 \%$ com sobrepeso e $11,2 \%$ com obesidade e $89,6 \%$ $(\mathrm{n}=320)$ dos alunos afirmaram não possuir nenhuma doença. Em relação à autopercepção do consumo alimentar, 198 (55,5\%) estudantes não consideram a própria alimentação saudável e 295 $(82,6 \%)$ acreditam que precisam realizar mudanças na própria alimentação. Quanto às fontes 
Quadro 1. Descrição dos Dez Passos para uma Alimentação Adequada e Saudável e sumário dos critérios utilizados para considerar que um passo foi realizado.

\begin{tabular}{|c|c|c|c|}
\hline \multicolumn{2}{|r|}{ Passos } & \multirow[b]{2}{*}{\begin{tabular}{l}
\multicolumn{1}{c}{ Componentes do passo } \\
Quantas vezes por semana você \\
costuma almoçar (arroz, feijão, carne \\
e salada por exemplo)? \\
Quantas vezes por semana você \\
consome frutas, verduras ou \\
legumes?
\end{tabular}} & \multirow[b]{2}{*}{\begin{tabular}{|l|}
\multicolumn{1}{|c|}{ Realização do passo } \\
Almoçar (arroz, feijão, carne \\
e salada) todos os dias da \\
semana; \\
Consumir frutas, verduras \\
ou legumes todos os dias da \\
semana.
\end{tabular}} \\
\hline Passo 1 & $\begin{array}{l}\text { Fazer de alimentos in } \\
\text { natura ou minimamente } \\
\text { processados a base da } \\
\text { alimentação. }\end{array}$ & & \\
\hline Passo 2 & $\begin{array}{l}\text { Utilizar óleos, gorduras, } \\
\text { sal e açúcar em pequenas } \\
\text { quantidades ao temperar e } \\
\text { cozinhar alimentos e criar } \\
\text { preparações culinárias. } \\
\end{array}$ & $\begin{array}{l}\text { Você costuma adicionar sal ou } \\
\text { açúcar extra aos alimentos ou } \\
\text { preparações (ex. sal no seu prato de } \\
\text { almoço, açúcar em um suco, etc.)? }\end{array}$ & $\begin{array}{l}\text { Não adicionar sal ou açúcar } \\
\text { extra aos alimentos ou } \\
\text { preparações. }\end{array}$ \\
\hline Passo 3 & $\begin{array}{l}\text { Limitar o consumo de } \\
\text { alimentos processados. }\end{array}$ & $\begin{array}{l}\text { Quantas vezes por semana você } \\
\text { consome cereais ou alimentos } \\
\text { integrais (pão integral, arroz integral, } \\
\text { biscoito integral, etc.)? }\end{array}$ & $\begin{array}{l}\text { Consumir cereais ou alimentos } \\
\text { integrais } 4 \text { vezes ou menos por } \\
\text { semana. }\end{array}$ \\
\hline Passo 4 & $\begin{array}{l}\text { Evitar o consumo } \\
\text { de alimentos } \\
\text { ultraprocessados. }\end{array}$ & $\begin{array}{l}\text { Quando você consome bebida junto } \\
\text { com a refeição, qual você costuma } \\
\text { escolher? } \\
\text { Quantas vezes por semana você } \\
\text { costuma comer salgadinhos } \\
\text { de pacote, biscoitos recheados, } \\
\text { macarrão instantâneo ou chocolates? }\end{array}$ & $\begin{array}{l}\text { Não consumir suco de pacote } \\
\text { ou de garrafa ou refrigerante } \\
\text { junto com as refeições; } \\
\text { e } \\
\text { Restringir o consumo de } \\
\text { alimentos ultraprocessados } \\
\text { para }<2 \text { vezes na semana. }\end{array}$ \\
\hline Passo 5 & $\begin{array}{l}\text { Comer com regularidade } \\
\text { e atenção, em ambientes } \\
\text { apropriados e, sempre que } \\
\text { possível, com companhia. }\end{array}$ & $\begin{array}{l}\text { Você costuma fazer as refeições } \\
\text { acompanhado(a) da família, amigos } \\
\text { ou colegas? } \\
\text { Quantas refeições você costuma fazer } \\
\text { por dia, incluindo lanches? } \\
\text { Você costuma fazer as refeições } \\
\text { assistindo TV, lendo ou manuseando } \\
\text { celular ou outros aparelhos } \\
\text { eletrônicos? }\end{array}$ & $\begin{array}{l}\text { Realizar as refeições sempre } \\
\text { acompanhado(a); } \\
\text { Realizar no mínimo três } \\
\text { refeições por dia; } \\
\text { Nunca fazer as refeições } \\
\text { assistindo TV, lendo ou } \\
\text { manuseando celular ou outros } \\
\text { aparelhos eletrônicos }\end{array}$ \\
\hline Passo 6 & \begin{tabular}{|l|} 
Fazer compras em locais \\
que ofertem variedades \\
de alimentos in natura ou \\
minimamente processados.
\end{tabular} & $\begin{array}{l}\text { Você ou sua família costumam fazer } \\
\text { compras em feiras agroecológicas de } \\
\text { alimentos? }\end{array}$ & $\begin{array}{l}\text { Realizar compras em feiras } \\
\text { agroecológicas uma vez por } \\
\text { semana. }\end{array}$ \\
\hline Passo 7 & $\begin{array}{l}\text { Desenvolver, exercitar } \\
\text { e partilhar habilidades } \\
\text { culinárias. }\end{array}$ & Quem prepara suas refeições? & $\begin{array}{l}\text { Preparar suas próprias } \\
\text { refeições. }\end{array}$ \\
\hline Passo 8 & $\begin{array}{l}\text { Planejar o uso do tempo } \\
\text { para dar à alimentação o } \\
\text { espaço que ela merece. }\end{array}$ & $\begin{array}{l}\text { Como você costuma fazer as } \\
\text { refeições? } \\
\text { Você costuma fazer as refeições } \\
\text { assistindo TV, lendo ou manuseando } \\
\text { celular ou outros aparelhos } \\
\text { eletrônicos? }\end{array}$ & $\begin{array}{l}\text { Realizar as refeições com } \\
\text { tranquilidade; } \\
\text { Nunca fazer as refeições } \\
\text { assistindo TV, lendo ou } \\
\text { manuseando celular ou outros } \\
\text { aparelhos eletrônicos }\end{array}$ \\
\hline Passo 9 & $\begin{array}{l}\text { Dar preferência, quando } \\
\text { fora de casa, a locais que } \\
\text { servem refeições feitas na } \\
\text { hora. }\end{array}$ & $\begin{array}{l}\text { Em um dia comum fora do lar, qual } \\
\text { estabelecimento você escolhe para } \\
\text { fazer suas refeições? }\end{array}$ & $\begin{array}{l}\text { Realizar as refeições em } \\
\text { restaurantes a quilo quando } \\
\text { fora de casa. }\end{array}$ \\
\hline Passo 10 & \begin{tabular}{|l|} 
Ser crítico quanto a \\
informações, orientações \\
e mensagens sobre \\
alimentação veiculadas em \\
propagandas comerciais.
\end{tabular} & $\begin{array}{l}\text { Quando você se depara com } \\
\text { uma informação relacionada à } \\
\text { alimentação e/ou nutrição, o que } \\
\text { você faz? }\end{array}$ & $\begin{array}{l}\text { Buscar fontes confiáveis ao } \\
\text { se deparar com informações } \\
\text { relacionadas à alimentação e/ } \\
\text { ou nutrição. }\end{array}$ \\
\hline
\end{tabular}


Tabela 1. Dados sociodemográficos, classificação antropométrica e de saúde dos universitários. Porto Alegre, RS, Brasil, 2018 ( $\mathrm{n}=357)$.

\begin{tabular}{|c|c|c|}
\hline Variáveis & n & $\%$ \\
\hline \multicolumn{3}{|l|}{ Sexo } \\
\hline Feminino & 219 & 61,3 \\
\hline \multicolumn{3}{|l|}{ Idade } \\
\hline$\leq 19$ anos & 98 & 27,5 \\
\hline 20 a 29 anos & 200 & 56,0 \\
\hline$\geq 30$ anos & 59 & 16,5 \\
\hline \multicolumn{3}{|l|}{ Estado Civil } \\
\hline Solteiro (a) & 300 & 84,0 \\
\hline Casado (a) & 52 & 14,6 \\
\hline Divorciado (a) & 5 & 1,4 \\
\hline \multicolumn{3}{|l|}{ Curso } \\
\hline Ciências da saúde & 79 & 22,1 \\
\hline Ciências humanas & 97 & 27,2 \\
\hline Ciências exatas e da terra & 6 & 1,7 \\
\hline Engenharias & 41 & 11,5 \\
\hline Indústria criativa & 66 & 18,5 \\
\hline Ciências sociais aplicadas & 68 & 19,0 \\
\hline \multicolumn{3}{|l|}{ Semestre } \\
\hline Início do curso & 223 & 62,5 \\
\hline \multicolumn{3}{|l|}{ Classificação antropométrica } \\
\hline Desnutrição & 16 & 4,5 \\
\hline Eutrofia & 219 & 61,3 \\
\hline Sobrepeso & 82 & 23,0 \\
\hline Obesidade & 40 & 11,2 \\
\hline \multicolumn{3}{|l|}{ Doença prévia } \\
\hline Obesidade & 21 & 5,9 \\
\hline Diabetes mellitus & 2 & 0,6 \\
\hline Hipertensão arterial & 5 & 1,4 \\
\hline Hipercolesterolemia & 9 & 2,5 \\
\hline Não possuo & 320 & 89,6 \\
\hline \multicolumn{3}{|l|}{ Considera alimentação saudável } \\
\hline Não & 198 & 55,5 \\
\hline \multicolumn{3}{|l|}{$\begin{array}{l}\text { Acredita que precisa fazer mudanças na } \\
\text { alimentação }\end{array}$} \\
\hline Sim & 295 & 82,6 \\
\hline \multicolumn{3}{|l|}{$\begin{array}{l}\text { Acesso à informação relacionada à } \\
\text { nutrição }\end{array}$} \\
\hline Mídia, TV, Internet & 213 & 59,7 \\
\hline Revistas & 1 & 0,3 \\
\hline Família, amigos & 27 & 7,6 \\
\hline Médico & 15 & 4,2 \\
\hline Educador físico & 4 & 1,1 \\
\hline Nutricionista & 84 & 23,5 \\
\hline $\begin{array}{l}\text { Guia Alimentar para a População } \\
\text { Brasileira }\end{array}$ & 1 & 0,3 \\
\hline Outro & 12 & 3,4 \\
\hline
\end{tabular}

de conhecimentos relacionados à alimentação $\mathrm{e}$ nutrição, destacam-se mídia, TV e internet com $59,7 \%$.

A seguir, na Tabela 2 está descrita a porcentagem de adesão a cada passo do Guia Alimentar. Nota-se que apenas os passos P2 e P3 do Guia Alimentar demonstraram adesão maior que $60 \%$.

A frequência de adesão aos passos do Guia Alimentar foi categorizada de acordo com as variáveis sociodemográficas analisadas (Tabela 3). $\mathrm{O}$ sexo feminino e a idade estiveram diretamente relacionados aos passos $\mathrm{P} 7$ e $\mathrm{P} 10$, os quais preconizam: desenvolver, exercitar e partilhar habilidades culinárias e ser crítico quanto a informações, orientações e mensagens sobre alimentação veiculadas em propagandas comerciais, respectivamente.

Tabela 2. Adesão aos Dez Passos para uma Alimentação Adequada e Saudável do Guia Alimentar para a População Brasileira por estudantes universitários do RS.

\begin{tabular}{|c|c|c|}
\hline \multirow{2}{*}{ Passos } & \multicolumn{2}{|c|}{ Adesão } \\
\hline & $\mathbf{n}$ & $\%$ \\
\hline $\begin{array}{l}\text { 1. Fazer de alimentos in natura ou } \\
\text { minimamente processados a base da } \\
\text { alimentação. }\end{array}$ & 65 & 18,2 \\
\hline $\begin{array}{l}\text { 2. Utilizar óleos, gorduras, sal e } \\
\text { açúcar em pequenas quantidades ao } \\
\text { temperar e cozinhar alimentos e criar } \\
\text { preparações culinárias. }\end{array}$ & 229 & 64,1 \\
\hline $\begin{array}{l}\text { 3. Limitar o consumo de alimentos } \\
\text { processados. }\end{array}$ & 283 & 79,3 \\
\hline $\begin{array}{l}\text { 4. Evitar o consumo de alimentos } \\
\text { ultraprocessados. }\end{array}$ & 154 & 43,1 \\
\hline $\begin{array}{l}\text { 5. Comer com regularidade e atenção, } \\
\text { em ambientes apropriados e, sempre } \\
\text { que possível, com companhia. }\end{array}$ & 27 & 7,6 \\
\hline $\begin{array}{l}\text { 6. Fazer compras em locais que } \\
\text { ofertem variedades de alimentos in } \\
\text { natura ou minimamente processados. }\end{array}$ & 49 & 13,7 \\
\hline $\begin{array}{l}\text { 7. Desenvolver, exercitar e partilhar } \\
\text { habilidades culinárias. }\end{array}$ & 108 & 30,3 \\
\hline $\begin{array}{l}\text { 8. Planejar o uso do tempo para dar à } \\
\text { alimentação o espaço que ela merece. }\end{array}$ & 25 & 7,0 \\
\hline $\begin{array}{l}\text { 9. Dar preferência, quando fora de } \\
\text { casa, a locais que servem refeições } \\
\text { feitas na hora. }\end{array}$ & 190 & 53,2 \\
\hline $\begin{array}{l}\text { 10. Ser crítico quanto a informações, } \\
\text { orientações e mensagens sobre } \\
\text { alimentação veiculadas em } \\
\text { propagandas comerciais. }\end{array}$ & 199 & 55,7 \\
\hline
\end{tabular}

Fonte: Elaborado pela autora. 


\begin{tabular}{|c|c|c|c|c|c|c|c|c|c|c|c|}
\hline \multirow[t]{23}{*}{ 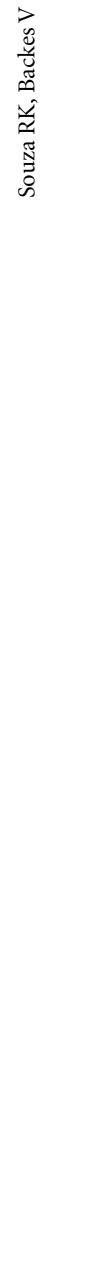 } & & \multicolumn{9}{|c|}{$\begin{array}{l}\text { Tabela 3. Frequência de adesão aos Dez Passos para uma Alimentação Adequada e Saudável do Guia Alimentar } \\
\text { para a População Brasileira por estudantes universitários do RS segundo as variáveis sociodemográficas } \\
\text { analisadas. }\end{array}$} & ntar \\
\hline & Variáveis & P1 & P2 & P3 & P4 & P5 & P6 & P7 & P8 & P9 & P10 \\
\hline & Sexo & & & & & & & * & & & $* *$ \\
\hline & Masculino & 15,9 & 65,2 & 79,7 & 47,1 & 5,8 & 12,3 & 22,5 & 4,3 & 47,8 & 43,5 \\
\hline & Feminino & 19,6 & 63,5 & 79,0 & 40,6 & 8,7 & 14,6 & 35,2 & 8,7 & 53,2 & 63,5 \\
\hline & Idade & & & & & & & * & & & ** \\
\hline & $\leq 19$ anos & 16,3 & 60,2 & 83,7 & 41,8 & 7,1 & 10,2 & 17,3 & 8,2 & 55,1 & 42,9 \\
\hline & 20 a 29 anos & 18,0 & 66,5 & 77,5 & 44,5 & 7,0 & 15,0 & 34,0 & 5,5 & 50,5 & 55,0 \\
\hline & $\geq 30$ anos & 22,0 & 62,7 & 78,0 & 40,7 & 10,2 & 15,3 & 39,0 & 10,2 & 59,3 & 79,7 \\
\hline & Estado Civil & & & & & & & ** & & * & \\
\hline & Solteiro (a) & 17,7 & 62,0 & 80,7 & 43,3 & 6,3 & 14,3 & 26,3 & 6,3 & 52,7 & 53,7 \\
\hline & Casado (a) & 17,3 & 75,0 & 75,0 & 42,3 & 15,4 & 9,6 & 50,0 & 11,5 & 61,5 & 65,4 \\
\hline & Divorciado (a) & 60,0 & 80,0 & 40,0 & 40,0 & 0,0 & 20,0 & 60,0 & 0,0 & 0,0 & 80,0 \\
\hline & Curso & & & & & & * & & & & * \\
\hline & Ciências da saúde & 16,5 & 67,1 & 79,7 & 43,0 & 5,1 & 8,9 & 43,0 & 1,3 & 49,4 & 72,2 \\
\hline & Ciências humanas & 23,7 & 62,9 & 77,3 & 40,2 & 11,3 & 18,6 & 27,8 & 9,3 & 64,9 & 51,5 \\
\hline & $\begin{array}{l}\text { Ciências exatas e da } \\
\text { terra }\end{array}$ & 0,0 & 33,3 & 83,3 & 33,3 & 0,0 & 0,0 & 33,3 & 0,0 & 50,0 & 50,0 \\
\hline & Engenharias & 14,6 & 68,3 & 75,6 & 51,2 & 9,8 & 9,8 & 26,8 & 12,5 & 36,6 & 48,8 \\
\hline & Indústria criativa & 16,7 & 62,1 & 80,3 & 43,9 & 3,0 & 22,7 & 27,3 & 7,6 & 51,5 & 48,5 \\
\hline & $\begin{array}{l}\text { Ciências sociais } \\
\text { aplicadas }\end{array}$ & 17,6 & 64,7 & 82,4 & 42,6 & 8,8 & 7,4 & 23,5 & 7,4 & 52,9 & 54,4 \\
\hline & Semestre & & & & & & & * & & & * \\
\hline & Início do curso & 19,3 & 61,0 & 82,1 & 47,1 & 8,1 & 12,6 & 24,7 & 8,5 & 52,9 & 50,2 \\
\hline & Final do curso & 16,4 & 69,4 & 74,6 & 36,6 & 6,7 & 15,7 & 39,6 & 4,5 & 53,7 & 64,9 \\
\hline
\end{tabular}

Nota: Teste de Qui-quadrado de Pearson; Tendência Linear; ${ }^{*} \mathrm{p}<0,05 ;{ }^{* *} \mathrm{p}<0,001$.

O estado civil esteve estatisticamente associado a dois dos dez passos, sendo que solteiros apresentaram menor frequência de realização do passo P7 (exercício de habilidades culinárias) quando comparados com casados e divorciados $(\mathrm{p}<0,001)$ e os casados realizavam com maior frequência o passo $\mathrm{P} 9$, que preconiza a preferência, quando fora de casa, a locais que servem refeições feitas na hora, quando comparados aos solteiros e divorciados $(\mathrm{p}<0,05)$.

Em relação aos cursos notou-se que o passo P6, cujo preconiza a realização das compras em locais que oferecem variedades de alimentos in natura ou minimamente processados, esteve significativamente relacionado aos cursos da indústria criativa. O passo $\mathrm{P} 10$, cujo orienta a ser crítico quanto a informações sobre alimentação veiculadas em propagandas comerciais, relacionou-se aos cursos da área de ciências da saúde.
Os passos P7 e P10 demonstraram relação direta com os estudantes que cursavam o final curso.

Na Tabela 4 está descrita a frequência de adesão aos Dez Passos para uma Alimentação Adequada e Saudável segundo classificação antropométrica e autopercepção do consumo alimentar. Quanto à classificação antropométrica, apenas o passo $\mathrm{P} 9$, que preconiza a escolha de locais que servem refeições feitas na hora, esteve estatisticamente associado, sendo que entre os que realizavam o passo, $57,9 \%$ não possuem excesso de peso ( $\mathrm{p}<0,05)$. A autopercepção alimentar positiva demonstrou relação estatisticamente significante entre os passos P1, P2, P3, P4, P6, P7, P8 e P10. Dentre estes, os passos P1, P4, P6, P7 e P8, demonstraram porcentagens de adesão inferiores a $60 \%$, diferentemente dos passos $\mathrm{P} 2$, P3 e P10 com prevalência de adesão de $72,3 \%, 64,2 \%$ e $69,2 \%$, respectivamente. 


\section{Discussão}

Observou-se uma baixa adesão às recomendações para a maioria dos passos. Estes dados são preocupantes uma vez que a má alimentação é um dos determinantes da obesidade, doença esta que atinge milhões de pessoas no mundo ${ }^{15}$. $\mathrm{O}$ atual estudo avaliou universitários de 18 a 67 anos de uma universidade privada da região sul do Brasil e encontrou prevalência de $34,2 \%$ de excesso de peso, resultado ainda maior que o encontrado por outros autores. Feitosa et al. ${ }^{3}$ verificaram em análise realizada com 718 estudantes de graduação da Universidade Federal do Sergipe, de diversas áreas, que $17,9 \%$ dos estudantes apresentavam excesso de peso. Pires e Mussi ${ }^{4}$ encontraram prevalência de mais de 30\% de excesso de peso entre 154 graduandas do curso de enfermagem em uma universidade na Bahia.

$\mathrm{O}$ estudo também encontrou baixa adesão aos Dez Passos para uma Alimentação Adequada e Saudável do Guia Alimentar ao constatar que oito dos dez passos demonstraram uma adesão inferior a $60 \%$, evidenciando que os estudantes possuem uma alimentação inadequada. De forma semelhante, Perez et al. ${ }^{16}$ analisaram os hábitos alimentares de 1.336 estudantes ingressantes em uma universidade do Rio de Janeiro e observaram baixo consumo diário de alimentos in natura como feijão, hortaliças e frutas e maior consumo diário de bebidas açucaradas, guloseimas e biscoitos ou salgadinhos de pacote. Analisando os dados da Pesquisa Nacional de Saúde (2013), Claro et al..$^{17}$ verificaram um consumo maior de doces entre adultos jovens, entre populações urbanas e na região sul do Brasil. Apesar do presente estudo não avaliar o consumo dos grupos alimentares, é possível perceber o baixo consumo de alimentos in natura através da baixa adesão ao primeiro passo do Guia Alimentar, o baixo con- sumo de carboidratos complexos por meio do passo 3 e alto consumo de alimentos ultraprocessados mediante a baixa adesão ao passo 4 .

Analisando a adesão aos passos do Guia Alimentar, nota-se alguns passos que apresentaram os menores percentuais de adesão: P1 - Fazer de alimentos in natura ou minimamente processados a base da alimentação; P5 - Comer com regularidade e atenção, em ambientes apropriados e, sempre que possível, com companhia; P6 - Fazer compras em locais que ofertem variedades de alimentos in natura ou minimamente processados; P8 - Planejar o uso do tempo para dar à alimentação o espaço que ela merece. $O$ passo P1 representa o baixo consumo de alimentos in natura e minimamente processados tais como frutas, legumes, verduras, grãos, etc, que corroboram com os estudos realizados por Moreira et al. ${ }^{18}$ e Loureiro et al. ${ }^{19}$. Já os passos P5, P6 e P8 estão mais relacionados com o planejamento do uso do tempo, o que pode estar associado à rotina de estudos dos alunos. Segundo Busato et al. ${ }^{20}$ o comportamento alimentar dos jovens sofre grande influência do ingresso na universidade, pois muitos ao sair de casa para estudar, passam a morar sozinhos e precisam cuidar da própria alimentação. Com o deslocamento para a universidade e as tarefas acadêmicas é comum não sobrar tempo para preparar um lanche saudável, então opta-se por lanches rápidos muitas vezes calóricos e pouco nutritivos. O que também é evidenciado devido a maior parcela dos alunos (62,5\%) estar cursando o início do curso, podendo ser ainda uma fase de adaptação.

As variáveis sexo feminino, idade maior ou igual a 30 anos e estar cursando o final do curso estiveram relacionadas aos passos $\mathrm{P} 7(\mathrm{p}<0,05)$ e P10 ( $<<0,001)$ do Guia Alimentar, que preconizam o exercício de habilidades culinárias e o olhar crítico diante das informações, orientações 
e mensagens sobre alimentação veiculadas em propagandas comerciais, respectivamente. A relação com o sexo feminino pode ser associada ao fato de que a mulher é incentivada a desenvolver habilidades culinárias ${ }^{21}$ e consequentemente possui uma preocupação maior com a saúde e mais interesse por assuntos que envolvem alimentação ${ }^{22,23}$. A fase de adaptação e o enfrentamento de algumas dificuldades no que se refere a adoção de uma alimentação saudável, podem explicar a relação entre o exercício de habilidades culinárias e o olhar mais crítico com idade maior ou igual a 30 anos e estar cursando o final do curso ${ }^{20}$.

Outra conduta avaliada neste estudo foi a atitude diante da compra de alimentos, por meio da qual notou-se que o aspecto mais relevante citado pelos estudantes foi a qualidade (35\%), sendo a informação nutricional a menos citada $(13,44 \%)$. Resultados semelhantes foram encontrados por Lopez-Cano et al. ${ }^{24}$, os quais constataram que adultos predominantemente de alta escolaridade realizam as compras de alimentos com base em tradição, valor e sabor. No mesmo estudo, os autores verificaram que apenas 49\% dos entrevistados afirmou ler o rótulo e que entre estes, as informações mais consultadas eram as calorias e as gorduras, demonstrando baixa busca pela qualidade nutricional do alimento. A fonte de informação relacionada à alimentação e nutrição mais citada pelos alunos foi mídia, TV e internet $(59,7 \%)$. A mídia e a internet exercem um grande poder sobre as escolhas alimentares, o qual geralmente é negativo ${ }^{25,26}$. Portanto, é necessária a veiculação correta de informações nutricionais através da mídia, TV ou internet pois além de ser a principal fonte de informação relacionada à alimentação, soma-se ao aspecto de $44,2 \%$ dos estudantes não buscarem fontes con-



Quando analisada a frequência de adesão aos passos do Guia Alimentar de acordo com a classificação antropométrica, observou-se significância estatística apenas em relação ao passo P9 (p $<0,05)$, demonstrando que quando fora de casa, os alunos sem excesso de peso optam mais por locais que servem refeições feitas na hora do que alunos com excesso de peso.

Um achado importante do presente estudo foi em relação à autopercepção do consumo alimentar e adesão aos Dez Passos para uma Alimentação Adequada e Saudável. De acordo com os dados coletados, dentre os estudantes que não consideram a alimentação saudável, encontrouse adesão superior a $60 \%$ para apenas um passo. Porém, observando os que consideram a alimen- tação saudável, apenas três dos dez passos obtiveram uma adesão de mais de $60 \%$. Isso demonstra que os estudantes provavelmente não possuem uma visão adequada da própria alimentação. Esse resultado corrobora com o sugerido por outros autores que avaliaram a percepção acerca da alimentação e concluíram que a dificuldade para a mudança de comportamentos alimentares não saudáveis está na concepção incorreta da própria alimentação, conferindo a falsa sensação de que não há necessidade de mudanças na dieta ${ }^{7-9}$.

Além da autopercepção alimentar, foi possível notar uma distorção da percepção de saúde entre os estudantes obesos. De acordo com a classificação do IMC, 11,25\% dos alunos são obesos, no entanto apenas 5,9\% declararam-se como tal. Percepções mais negativas da saúde estão associadas à idade, à escolaridade ${ }^{29} \mathrm{e}$ a características funcionais limitadas ${ }^{30}$, o que pode explicar o fato de os estudantes, por serem jovens, cursarem nível superior e não possuírem limitações físicas, respectivamente, não associarem a obesidade a uma doença.

O presente estudo apresenta algumas limitações metodológicas que podem interferir nos resultados, como por exemplo, o questionário utilizado para a coleta dos dados. Apesar de passar por um pré-teste, o instrumento utilizado no presente estudo não foi validado, sendo portanto, suscetível a viés diante dos resultados obtidos, como é o caso da alta adesão ao passo 3 por exemplo, que pode estar mascarada pelo baixo consumo de alimentos integrais pelos alunos. Outro possível viés está relacionado aos dados antropométricos dos estudantes, que não foram mensurados. Apesar da possibilidade de serem subestimados ou superestimados, estudos prévios mostram que os dados de peso e altura referidos são confiáveis, pois demonstram baixa variação em relação aos mesmos dados quando mensurados ${ }^{31,32}$. Por ser um estudo de delineamento transversal, não é possível chegar a conclusões, realizando-se apenas associações entre os resultados ${ }^{33}$. Tratandose de uma amostra por conveniência, incluiu-se estudantes de uma única universidade privada do sul do país, não permitindo-se extrapolar os dados para alunos de outras universidades. Apesar de não contemplar todos os universitários do campus, a amostra estudada incluiu estudantes de todos os cursos, abrangendo as diferentes formações profissionais ${ }^{34}$.

O Guia Alimentar é um instrumento atual e ainda pouco utilizado em estudos que avaliam o comportamento alimentar. Suas recomendações preconizam o resgate da alimentação baseada em 
alimentos in natura ou minimamente processados, classificando os alimentos de acordo com o seu grau de processamento e não conforme os grupos alimentares ${ }^{6}$. Portanto, é importante salientar a originalidade do estudo, por ser o primeiro a propor uma avaliação da adesão às recomendações do novo Guia Alimentar.

\section{Conclusão}

Com base nas novas diretrizes alimentares preconizadas pelo Guia Alimentar, o estudo demonstrou prevalência de inadequação da alimentação dos estudantes, assim como alta prevalência de excesso de peso. Os estudantes que consideraram a própria alimentação saudável demonstraram uma percepção distorcida da mesma, o que pôde ser observado através da baixa adesão aos passos do Guia Alimentar. Esses resultados sinalizam para uma concepção imprecisa da própria alimentação, dificultando possíveis mudanças de comportamentos alimentares inadequados, uma vez que quando não reconhecida a necessidade de mudanças, os comportamentos tendem a permanecer inalterados. A principal fonte de informação nutricional citada pelos estudantes foi mídia, TV e internet, o que exige um olhar atento e crítico. No entanto, pouco mais da metade dos estudantes afirmou buscar fontes confiáveis quando deparados a informações sobre alimentação e nutrição. Planejar o uso do tempo, comer com regularidade e atenção, partilhar habilidades culinárias, preferir alimentos in natura e fazer compras em feiras agroecológicas parecem ser os maiores desafios enfrentados por essa população.

Considerando-se que o comportamento alimentar envolve diversos aspectos comportamentais e sociais muito além do ato de consumir alimentos, são necessárias ações de educação alimentar e nutricional voltadas para essa população, a fim de possibilitar autonomia alimentar, auxiliando na solução dos problemas alimentares decorrentes da rotina de estudos. Mais estudos são necessários para avaliar a autopercepção do consumo alimentar da população, uma vez que o reconhecimento das inadequações alimentares é o primeiro passo para a realização de mudanças no comportamento alimentar.

\section{Colaboradores}

As autoras declaram que participaram da construção e formação deste estudo. RK Souza participou da concepção, redação do artigo e análise de dados e $\mathrm{V}$ Backes contribuiu para a análise de dados e revisão da versão final a ser publicada.

\section{Referências}

1. Toral N, Slater B. Abordagem do modelo transteórico no comportamento alimentar. Cien Saude Colet 2007; 12(6):1641-1650.

2. Alvarenga M, Figueiredo M, Timerman F, Antonaccio C. Nutrição Comportamental. Barueri: Manole; 2016.

3. Feitosa EPS, Dantas CAO, Andrade-Wartha ERS, Marcellini PS, Mendes-Netto RS. Hábitos alimentares de estudantes de uma universidade pública no nordeste, Brasil. Alim Nutr 2010; 21(2):225-230.

4. Pires CGS, Mussi FC. Excesso de peso em universitários ingressantes e concluintes de um curso de enfermagem. Esc Anna Nery 2016; 20(4):1-5.

5. Barbosa LB, Vasconcelos SML, Correia LOS, Ferreira RC. Estudos de avaliação do conhecimento nutricional de adultos: uma revisão sistemática. Cien Saude Colet 2016; 21(2):449-462.

6. Brasil. Ministério da Saúde (MS). Secretaria de Atenção à Saúde. Departamento de Atenção Básica. Guia alimentar para a população brasileira. $2^{\text {a }}$ ed. Brasília: MS; 2014.

7. Kearney M, Gibney MJ, Martinez JÁ, Almeida MD, Friebe D, Zunft HJ, Widhalm K, Kearney JM, 1997 apud Toral N, Slater B. Abordagem do modelo transteórico no comportamento alimentar. Cien Saude Colet 2007; 12(6):1641-1650. 
8. Biloukha O, Utermohlen V, 2001 apud Toral N, Slater B. Abordagem do modelo transteórico no comportamento alimentar. Cien Saude Colet 2007; 12(6):16411650.

9. Reid DJ, Conrad SA, Hendricks SM, 1996 apud Toral N, Slater B. Abordagem do modelo transteórico no comportamento alimentar. Cien Saude Colet 2007; 12(6):1641-1650.

10. Conselho Nacional de Desenvolvimento Científico e Tecnológico (CNPQ). Tabela de Áreas do Conhecimento [Internet]. Disponível em: http://www.cnpq. br/documents/10157/186158/TabeladeAreasdoConhecimento.pdf

11. World Health Organization (WHO). Global Database on Body Mass Index [Internet]. 2006. Disponível em: http://apps.who.int/bmi/index.jsp?introPage =intro_3.html

12. Brasil. Ministério da Saúde (MS). Departamento de Vigilância de Doenças e Agravos Não transmissíveis e Promoção da Saúde. Vigilância de fatores de risco e proteção para doenças crônicas por inquérito telefônico: estimativas sobre frequência e distribuição sociodemográfica de fatores de risco e proteção para doenças crônicas nas capitais dos 26 estados brasileiros e no Distrito Federal em 2016. Brasília: MS; 2017.

13. Ceccheto FH, Pellanda LC. Construção e validação de um questionário sobre conhecimento de hábitos saudáveis e fatores de risco para doenças cardiovasculares em estudantes. J Pediatr 2014; 90(4):415-419.

14. Crivellenti LC, Zuccolotto DCC, Sartorelli DS. Desenvolvimento de um Índice de Qualidade da Dieta Adaptado para Gestantes. Rev Saude Publica 2018; 52:59.

15. NCD Risk Factor Collaboration. Trends in adult body-mass index in 200 countries from 1975 to 2014: a pooled analysis of 1698 population-based measurement studies with $19 \cdot 2$ million participants. Lancet 2016; 387(10026):1377-1396.

16. Perez PMP, Castro IRR, Franco AS, Bandoni DH, Wolkoff DB. Práticas alimentares de estudantes cotistas e não cotistas de uma universidade pública brasileira. Cien Saude Colet 2016; 21(2):531-542.

17. Claro RM, Santos MAS, Oliveira TP, Pereira CA, Szwarcwald CL, Malta DC. Consumo de alimentos não saudáveis relacionados a doenças crônicas não transmissíveis no Brasil: Pesquisa Nacional de Saúde, 2013. Epidemiol Serv Saúde 2015; 24(2):257-265.

18. Moreira PRS, Rocha NP, Milagres LC, Novaes JF. Análise crítica da qualidade da dieta da população brasileira segundo o Índice de Alimentação Saudável: uma revisão sistemática. Cien Saude Colet 2015; 20(12):3907-3923.

19. Loureiro AS, Silva RMVG, Rodrigues PRM, Pereira RA, Wendpap LL, Ferreira MG. Qualidade da dieta de uma amostra de adultos de Cuiabá (MT): associação com fatores sociodemográficos. Rev Nutr 2013; 26(4):431-441.

20. Busato MA, Pedrolo C, Gallina LS, Rosa L. Ambiente e alimentação saudável: percepções e práticas de estudantes universitários. Semina 2015; 36(2):75-84.

21. Caldo P. Revistas, consumos, alimentación y saberes femininos. La propuesta de Damas y Damitas, Argentina, 1939-1944. Secuencia 2016; 94:210-239.
22. Almutairi KM, Alonazi WB, Vinluan MJ, Almigbal TH, Batais MA, Alodhayani AA, Alsadhan N, Tumala RB, Moussa M, Aboshaiqah AE, Alhoqail RI. Health promoting lifestyle of university students in Saudi Arabia: a cross-sectional assessment. BMC Public Health 2018; 18:1-10.

23. Faria YO, Gandolfi L, Moura LBA. Prevalência de comportamentos de risco em adulto jovem e universitário. Acta Paul Enferm 2014; 27(6):1-5.

24. López-Cano LA, Restrepo-Mesa SL, Medellín SS. Etiquetado nutricional, una mirada desde los consumidores de alimentos. Perspectivas Nutr Humana 2014; 16(2):145-158.

25. Moura TK, Fernandes MTM. A influência da mídia na alimentação: a moda do slow food. In: XVII Encontro Latino Americano de Iniciação Científica e XIII Encontro Latino Americano de Pós-Graduação. São José dos Campos: Universidade do Vale do Paraíba; 2013. p. 1-6.

26. Britto SR, Viebig RF, Morimoto JM. Analysis of food advertisements on cable television directed to children based on the food guide for the Brazilian population and current legislation. Rev Nutr 2016; 29(5):721-729.

27. Bairati L, Grasso E. Indicazioni in etichetta e messaggi fuorvianti nell'ínformazione del consumatore di alimenti. Rev Bio Der 2018; 42:33-49.

28. Osiac LR, Cofré C, Pizarro T, Mansilla C, Herrera CA, Burrows J, Castillo C. Using evidence-informed policies to tackle overweight and obesity in Chile. Rev Panam Salud Publica 2017; 41:1-5.

29. Silva VH, Rocha JSB, Caldeira AP. Fatores associados à percepção negativa de saúde em mulheres climatérias. Cien Saude Colet 2018; 23(5):1611-1620.

30. Zanesco C, Bordin D, Santos CB, Müller EV, Fadel CB. Factors determining the negative perception of the health of Brazilian elderly people. Rev Bras Geriatr Gerontol 2018; 21(3):283-292.

31. Moreira NF, Luz VG, Moreira CC, Pereira RS, Sichieri R, Ferreira MG, Muraro AP, Rodrigues PRM. Self-reported weight and height are valid measures to determine weight status: results from the Brazilian National Health Survey (PNS 2013). Cad Saúde Pública 2018; 34(5):1-13.

32. Lima LRM, Freitas RPA, Silva LRD, Medeiros ACQ. Estimation of body mass index from self-reported measures: what is the validity? J Phys Educ 2018; 29:19.

33. Fronteira I. Estudos Observacionais na Era da Medicina Baseada na Evidência: Breve Revisão Sobre a Sua Relevância, Taxonomia e Desenhos. Acta Med Port 2013; 26(2):161-170.

34. Valério RBC, Dutra FCMS. Envejecimiento funcional y capacidade de trabajo entre los trabajadores atendidos em la atención primaria. Cien Trab 2016; 18(57):190-195.

Artigo apresentado em 19/09/2018

Aprovado em 15/04/2019

Versão final apresentada em 17/04/2019 